\title{
OPTIMAL PILOT PLACEMENT FOR TIME-VARYING CHANNELS
}

\author{
Min Dong and Lang Tong \\ School of Electrical and Computer Engineering \\ Cornell University, Ithaca, NY 14853 \\ \{mdong,ltong\}@ece.cornell.edu
}

\author{
Brian M. Sadler \\ Army Research Laboratory \\ Adelphi, MD 20783 \\ bsadler@arl.army.mil
}

\begin{abstract}
Two major training techniques for wireless channels are the timedivision multiplexed (TDM) training and the superimposed training. For the TDM schemes with regular periodic placements (RPP), the closed-form expression of the steady-state minimum mean square error (MMSE) is obtained as a function of pilot placement for Gauss-Markov fading channels. We show that the single cluster RPP scheme (RPP-1) minimizes the maximum steady-state channel MMSE. For BPSK and QPSK signaling, we then show that the optimal placement that minimizes the maximum bit error rate (BER) is also RPP-1. We next compare the MMSE and BER performance under the superimposed training schemes with those under the optimal TDM scheme. It is shown that while RPP-1 scheme performs better at high SNR and for slow varying channels, the superimposed scheme outperforms RPP-1 in the other regimes. This demonstrates the potential for using superimposed training in relatively fast time-varying environments.
\end{abstract}

\section{INTRODUCTION}

Channel estimation is a major challenge for reliable wireless transmissions. Often in practice, pilot symbols known to the receiver are multiplexed with the data symbols for channel acquisition. Two major types of training for single carrier systems are the time division multiplexed (TDM) training and the superimposed training. Pilot symbols in a TDM system are inserted into the data stream under certain placement pattern, and the channel estimate is updated using these pilot symbols. For the superimposed training, on the other hand, pilot and data symbols are added and transmitted together, and the channel estimate is updated constantly. The way that pilot symbols are multiplexed into the data stream affects the system performance for time-varying channels. Under the TDM training, the presence of pilot symbols makes channel estimation accurate at some time and coarse at others. What is the optimal placement that minimizes the mean square error (MSE) of the channel estimator? Does the MSE-minimizing training also minimize the bit error rate (BER)? In choosing the optimal training scheme, do we need to know the rate of channel variation and the level of signal-to-noise ratio? How does TDM training compare with the superimposed training?

This work was supported in part by the Army Research Office under Grant ARO-DAAB19-00-1-0507, the Multidisciplinary University Research Initiative (MURI) under the Office of Naval Research Contract N00014-00-1-0564, and Army Research Laboratory CTA on Communication and Networks.
In this paper, we aim to address these issues systematically. We model the fading channel by a Gauss-Markov process and use the minimum mean square error (MMSE) channel estimator along with the symbol-by-symbol maximum likelihood (ML) detector. For the TDM training, we show that, among all periodic placements, the regular periodic placement with cluster size one (referred to as the RPP-1) minimizes both maximum channel MMSE and BER of BPSK and QPSK signaling, regardless of the level SNR or the rate of channel variation. Performance comparison between the optimal TDM scheme and the superimposed scheme is given numerically. We show that the TDM scheme performs better at high SNR and for slowly varying channels whereas the superimposed scheme is superior for many situations of practical importance. In the process of establishing the optimality of RPP1 , we also provide closed-form expressions of steady-state channel MMSE at each data symbol position, which is useful to evaluate the performance of coded transmissions.

Optimal training has been previously considered for block fading channels from a channel estimation perspective under both TDM and superimposed trainings $[9,5,2]$ and from an information theoretic angle in $[10,1]$. For time-varying channels, Cavers first analyzed the pilot symbol assisted modulation (PSAM) [3] that includes the periodic TDM training with cluster size one-the RPP-1 placement. While the optimality of RPP-1 has never been shown for either MMSE or BER until now, it has been applied in various settings. Furthermore, existing results tend to assume the RPP-1 placement and optimize parameters such as power and rate allocations [13, 8, 11]. In [4], we considered the problem of optimal placement of pilot symbols for packetized transmission over time varying channels at high SNR.

This paper is organized as follows. In Section 2, we optimize placement for the TDM schemes in terms of both channel tracking and BER performance. In Section 3, we derive the steady-state channel MSE for channel tracking and the bit error rate under the superimposed training. In Section 4, we provide the numerical performance comparison under the optimal TDM scheme and the superimposed scheme. Finally, we conclude in Section 5.

\section{OPTIMAL TDM TRAINING PLACEMENT}

\subsection{The Channel Model}

We model a time-varying flat Rayleigh fading channel given by

$$
y_{k}=s_{k} h_{k}+w_{k}, \quad k=1,2, \cdots
$$

where $y_{k}$ is the observation sequence, $s_{k}$ the transmitted symbols, $h_{k} \sim \mathcal{C} \mathcal{N}\left(0, \sigma_{h}^{2}\right)$ the zero mean complex Gaussian channel state 
with variance $\sigma_{h}^{2}$, and $w_{k} \stackrel{i . i . d .}{\sim} \mathcal{C N}\left(0, \sigma_{w}^{2}\right)$ is the complex circular AWGN at time $k$. We assume data $s_{k}$, channel $h_{k}$ and noise $w_{k}$ are independent.

The channel state $h_{k}$ is modeled by a first-order Gauss-Markov process

$$
h_{k}=a h_{k-1}+u_{k}, \quad u_{k} \stackrel{i . i . d .}{\sim} \mathcal{C N}\left(0,\left(1-a^{2}\right) \sigma_{h}^{2}\right)
$$

where $u_{k}$ is the white Gaussian driving noise. Parameter $a \in$ $[0,1]$ is the fading coefficient characterizing the degree of timevariation. The value of $a$ can be determined by the channel Doppler spread and the transmission bandwidth, where the relation among the three is found in [7]. The Gauss-Markov model has been widely adopted as a simple and effective model to characterize the fading process $[6,7,12]$.

\subsection{The Periodic TDM Placements}

We consider the class of periodic placements, as shown in Fig. 1, where the placement pattern of pilot symbols repeats periodically. The restriction to periodic placements is mild; a system with aperiodic training will not reach a steady state, and is seldom considered in practice. We define the period of a placement, denoted by $T$, to be the length of the smallest block over which the placement pattern repeats. Note that the starting point of a period can be arbitrarily chosen. Without loss of generality, we assume that each period starts with a pilot symbol and ends with a data symbol. In general, any periodic placement with $n$ clusters of pilot symbols in a period of length $T$ can be specified by a 2-tuple $\mathcal{P}=(\boldsymbol{\gamma}, \boldsymbol{\nu})$, where $\boldsymbol{\gamma}=\left[\gamma_{1}, \cdots, \gamma_{n}\right]$ is the pilot cluster length vector and $\boldsymbol{\nu}=\left[\nu_{1}, \cdots, \nu_{n}\right]$ the data block length vector, as illustrated in Fig. 2. Note that $T=\sum_{i}\left(\gamma_{i}+\nu_{i}\right)$. We further denote $\mathcal{I}_{p}(\mathcal{P})$ as the index set containing positions (relative to the beginning of the period) of the pilot symbols within one period. For different placement schemes, we assume the following:

A1) All pilot symbols have equal power, denoted by $\sigma_{p}^{2}$; the power for data symbols is denoted by $\sigma_{d}^{2}$.

A2) The percentage of pilot symbols in a data stream $\eta=\sum_{i} \gamma_{i} / T$ is fixed.

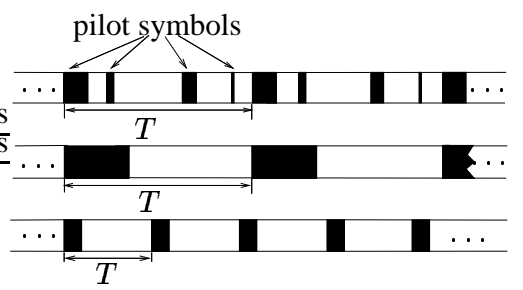

Fig. 1. Data streams with periodic placements

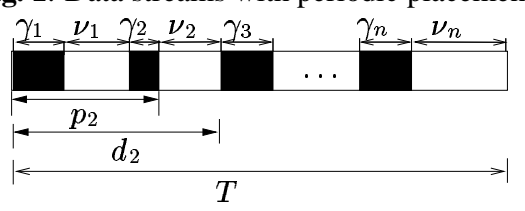

Fig. 2. Representation of placement within one placement period

\subsection{The Receiver}

We consider a typical receiver structure where the channel estimator provides the channel estimate $\hat{h}_{k}(\mathcal{P})$ to the demodulator, and the data symbol $s_{k}$ is detected based on the received sample $y_{k}$ and $\hat{h}_{k}(\mathcal{P})$ using the symbol-by-symbol ML detector.

For a given placement $\mathcal{P}$, the observations over pilot symbols are given by $\left\{y_{l T+k}: k \in \mathcal{I}_{p}(\mathcal{P}), l=0,1, \cdots\right\}$. We consider the MMSE channel estimator based on the current and all past pilot symbols and their corresponding observations. The MMSE channel estimate at time $(l T+k)$, denote by $\hat{h}_{l T+k}$ is given by

$$
\begin{aligned}
\hat{h}_{l T+k}(\mathcal{P})= & \mathrm{E}\left\{h_{l T+k} \mid\left\{y_{l T+j}: j \leq k, j \in \mathcal{I}_{p}(\mathcal{P}),\right.\right. \\
& \left.\left.y_{(l-m) T+j}: j \in \mathcal{I}_{p}(\mathcal{P}), m=1,2, \cdots\right\}\right\},
\end{aligned}
$$

which can be implemented recursively by Kalman filtering. The Kalman filter switches between two modes: it updates the channel estimate using pilot symbols during each training period and predicts the channel state during data transmission.

Given the channel estimate $\hat{h}_{k}$, the optimal detection is given by the ML detector. Conditioned on any data symbol $s_{k}, y_{k}$ and $\hat{h}_{k}$ are jointly Gaussian. For any phase-shift keying (PSK) signaling, we have $\left|s_{k}\right|^{2}=\sigma_{d}^{2}$, and the ML decision rule is given by

$$
\begin{aligned}
\hat{s}_{k} & =\underset{s_{k} \in\left\{\sigma_{d}^{2},-\sigma_{d}^{2}\right\}}{\arg \max } p\left(y_{k}, \hat{h}_{k} \mid s_{k}\right)=\arg \max _{s_{k}} \operatorname{Re}\left\{s_{k}^{*} \hat{h}_{k}^{*}(\mathcal{P}) y_{k}\right\} \\
& =\arg \min _{s_{k}}\left|y_{k}-\hat{h}_{k}(\mathcal{P}) s_{k}\right|^{2}
\end{aligned}
$$

which shows that the same ML detector for the known channel can be used by substituting the estimated channel.

\subsection{The Optimization Criteria}

Although for the long term behavior the system converges to a periodic steady state, within one placement period, the MSE and BER performance of TDM schemes are not stationary.

Given $\mathcal{P}$ with period $T$, let $M[l T+k ; \mathcal{P}]$ be the MMSE of channel estimate at time $(l T+k)$. During any training cluster, from the standard Kalman filter theory, we obtain the recursive expression for the MMSE as: for $k \in \mathcal{I}_{p}(\mathcal{P})$ and all integer $l$,

$M[l T+k ; \mathcal{P}]=\frac{\sigma_{w}^{2}\left(a^{2} M[l T+k-1 ; \mathcal{P}]+\left(1-a^{2}\right) \sigma_{h}^{2}\right)}{\sigma_{w}^{2}+\left(a^{2} M[l T+k-1 ; \mathcal{P}]+\left(1-a^{2}\right) \sigma_{h}^{2}\right) \sigma_{p}^{2}}$

Once the $i$ th training cluster in a placement period ends, of which the index (relative to the beginning of the period) is denoted by $p_{i}$ as shown in Fig.2, the Kalman filter predicts the channel state during data transmissions of duration $\nu_{i}$. The MMSE is given by

$M\left[l T+p_{i}+k ; \mathcal{P}\right]=a^{2 k} M\left[l T+p_{i} ; \mathcal{P}\right]+\sigma_{h}^{2}\left(1-a^{2 k}\right), k=1, \cdots, \nu_{i}$.

We are naturally interested in the steady state performance as $l \rightarrow$ $\infty$. Furthermore, we are only interested in the MSE of the channel estimates during data transmission. Let $M_{k}(\mathcal{P}) \triangleq \lim _{l \rightarrow \infty} M[l T+$ $k ; \mathcal{P}]$, then

$$
M_{p_{i}+k}(\mathcal{P})=a^{2 k} M_{p_{i}}(\mathcal{P})+\left(1-a^{2 k}\right) \sigma_{h}^{2}, k=1, \cdots, \nu_{i}
$$

which increases with $k$. Let $d_{i} \triangleq p_{i}+\nu_{i}$ be the index of the position of the last data symbol in the $i$ th block, as shown in Fig.2. Then, the maximum steady-state MMSE in this block is reached at $d_{i}$. The optimal placement that minimizes the maximum steady-state channel MMSE over data symbols during one placement period can then be obtained by

$$
\begin{aligned}
\mathcal{E}(\mathcal{P}) & =\max _{k: k \notin \mathcal{I}_{p}(\mathcal{P})} M_{k}(\mathcal{P})=\max _{1 \leq i \leq n} M_{d_{i}}(\mathcal{P}) \\
\mathcal{P}_{\text {MMSE }}^{*} & =\underset{\mathcal{P}}{\arg \min } \max _{1 \leq i \leq n} M_{d_{i}}(\mathcal{P}) .
\end{aligned}
$$


The BER performance is directly affected by channel MMSE, and our goal is to find the optimal placement that minimizes the maximum steady-state BER. Specifically, let $\mathrm{Pe}[k ; \mathcal{P}]$ be the steadystate BER at the $k$ th position of a period. We are interested in the following optimization

$$
\mathcal{P}_{\mathrm{BER}}^{*}=\arg \min _{\mathcal{P}} \max _{1 \leq k \leq T} \operatorname{Pe}[k ; \mathcal{P}]
$$

We show next that $\mathcal{P}_{\text {MMSE }}^{*}=\mathcal{P}_{\mathrm{BER}}^{*}$ for the BPSK and QPSK signaling.

Proposition 1 Under the Gauss-Markov channel model with BPSK or QPSK input symbols, if the MMSE channel estimator is used along with the ML detector, then

$$
\mathcal{P}_{M M S E}^{*}=\mathcal{P}_{B E R}^{*} .
$$

Proof: Define $\operatorname{snr}_{d} \triangleq \sigma_{d}^{2} / \sigma_{w}^{2}$. For BPSK, we can show that the BER for data symbols at the $k$ th position of a period is given by

$P_{e}[k ; \mathcal{P}]=\mathrm{E}\left\{Q\left(\sqrt{\frac{2\left|\hat{h}_{k}\right|^{2} \sigma_{d}^{2}}{\sigma_{d}^{2} M_{k}(\mathcal{P})+\sigma_{w}^{2}}}\right)\right\}=\frac{1}{2}\left[1-\sqrt{\frac{1-\frac{M_{k}(\mathcal{P})}{\sigma_{h}^{2}}}{1+\frac{1}{\sigma_{h}^{2} \operatorname{snr}_{d}}}}\right]$.

which is a function of the steady-state channel MMSE with placement $\mathcal{P}$. It immediately follows that, to optimize the placement in (5) is equivalent to that in (4). For QPSK, similar argument can be followed.

\subsection{The Optimal TDM Placement}

We first study the optimal placement for a special class of placements called regular periodic placement. The extension to the general class follows.

The regular periodic placement RPP- $\gamma$, defined as the placements satisfying $\mathcal{P}=(\gamma, \nu)$, has only one pilot cluster of size $\gamma$ and one data cluster of size $\nu$ with $T=\gamma / \eta$. In Fig. 1, the second and third examples are placements belonging to this class. From (3) and (4), it follows that for RPP- $\gamma$, the optimal placement is obtained by

$$
\gamma_{*}=\arg \min _{\gamma} \mathcal{E}(\gamma)=\arg \min _{\gamma} M_{T}(\gamma)
$$

Our problem now is to find the explicit expression of the steadystate solution $M_{T}(\gamma)$ and analyze its behavior as a function of pilot cluster size $\gamma$.

A useful quantity in the sequel is the steady state MMSE when all symbols are pilots, defined as $M_{\infty} \triangleq \lim _{\gamma \rightarrow \infty} M_{\gamma}(\gamma)$. Its expression can be obtained as the solution to the steady-state Riccati equation for the channel MMSE update. Due to the limited space, we won't elaborate here. The following Lemma provides the closed-form MMSE expression for the RPP- $\gamma$ placement.

Lemma 1 For any RPP- $\gamma$ scheme, the steady-state channel MMSE is given by

$$
\begin{aligned}
& M_{T}(\gamma)=\delta_{T}(\gamma)+M_{\infty} \\
& M_{k}(\gamma)=\frac{M_{T}(\gamma)-\sigma_{h}^{2}\left(1-a^{2(T-k)}\right)}{a^{2(T-k)}}, k=\gamma+1, \cdots, T-1
\end{aligned}
$$

where $\delta_{T}(\gamma)$ is computed by the following equations:

$\delta_{T}(\gamma)=-b_{\gamma}+\sqrt{b_{\gamma}^{2}+c_{\gamma}}$

$$
\begin{aligned}
& b_{\gamma} \triangleq\left(\frac{1-\left(\frac{a^{2 \frac{1-\eta}{\eta}}}{\alpha}\right)^{\gamma}}{1-\frac{1}{\alpha^{\gamma}}}\right)\left(\frac{\alpha-1}{2 \beta}\right)-\frac{\left(1-a^{2 \frac{1-\eta}{\eta} \gamma}\right)}{2} \rho_{\infty} \sigma_{h}^{2} \\
& c_{\gamma} \triangleq\left(\frac{1-a^{2 \frac{1-\eta}{\eta} \gamma}}{1-\frac{1}{\alpha^{\gamma}}}\right)\left(\frac{\alpha-1}{\beta}\right) \rho_{\infty} \sigma_{h}^{2}, \quad s n r_{p} \triangleq \frac{\sigma_{p}^{2}}{\sigma_{w}^{2}}, \rho_{\infty} \triangleq 1-\frac{M_{\infty}}{\sigma_{h}^{2}} \\
& \alpha \triangleq \frac{1}{a^{2}}\left(1+\left(1-a^{2} \rho_{\infty}\right) \sigma_{h}^{2} s n r_{p}\right)^{2}, \beta \triangleq s n r_{p}\left(1+\left(1-a^{2} \rho_{\infty}\right) \sigma_{h}^{2} s n r_{p}\right) .
\end{aligned}
$$

From (8), because $M_{\infty}$ is not a function of $\gamma$, the optimization in (7) can now be rewritten as

$$
\gamma_{*}=\underset{\gamma}{\arg \min } \delta_{T}(\gamma)
$$

By analyzing the behavior of $\delta_{T}(\gamma)$ as a function of $\gamma$, we obtain the optimal placement for RPP schemes in the following theorem.

Theorem 1 For the class of RPP placements, under A1-A2, the maximum MMSE of channel estimates during data transmission, $\mathcal{E}(\gamma)$, is a monotone increasing function of $\gamma$. Thus RPP-1 is optimal among all RPP placements, and the minimum $\mathcal{E}(\gamma)$ is given by

$$
\mathcal{E}^{T D M \triangleq} \min _{\gamma} \mathcal{E}(\gamma)=\mathcal{E}(1)=\sigma_{h}^{2}-a^{2 \frac{1-\eta}{\eta}}\left(\sigma_{h}^{2}-M_{1}\right)
$$

where

$$
M_{1}=\frac{\sigma_{h}^{2}}{\frac{1}{2}\left(1+\sigma_{h}^{2} s n r_{p}\right)+\sqrt{\left[\frac{1}{2}\left(1+\sigma_{h}^{2} s n r_{p}\right)\right]^{2}+\frac{a^{\frac{2}{\eta}}}{1-a^{\frac{2}{\eta}}} \sigma_{h}^{2} s n r_{p}}} .
$$

We next show that RPP-1 is in fact optimal among all periodic placements. Due to the limited space, we only outline a few steps required to prove the optimality of RPP-1. Consider first the case with two pilot clusters of lengths $\gamma=\left(\gamma_{1}, \gamma_{2}\right)$ and two data blocks of lengths $\boldsymbol{\nu}=\left(\nu_{1}, \nu_{2}\right)$. To minimize the maximum MMSE for the entire data blocks suggests the equalization rule that forces $M_{d_{1}}(\mathcal{P})=M_{d_{2}}(\mathcal{P})$, which leads to making pilot clusters equal, and eventually, results in the reduction to the RPP- $\gamma$ placement. This result can then be generalized to any $n$-cluster placement. Combining Theorem 1 and Proposition 1, we then have the optimality of RPP-1.

Theorem 2 Given a fixed percentage of pilot symbols $\eta$, the optimal placement for periodic TDM training that minimizes the maximum steady-state MMSE and BER for any first order Gauss-Markov channel is RPP-1.

We point out that this optimality holds regardless of the values of $\operatorname{snr}_{p}$ and $a$. Note also that our results immediately implies that if there is a constraint on the minimum cluster size $\gamma_{o}, \mathrm{RPP}-\gamma_{o}$ is optimal.

\section{THE SUPERIMPOSED TRAINING SCHEME}

We consider the time invariant power allocation where the transmitted symbol $s_{k}=\rho_{t} t_{k}+\rho_{d} d_{k}$ is the superposition of pilot and data symbols. The system equation in (1) is then given by $y_{k}=\left(\rho_{t} t_{k}+\rho_{d} d_{k}\right) h_{k}+w_{k}$, where $\left\{t_{k}\right\}$ is the pilot sequence and $\left\{d_{k}\right\}$ is the i.i.d. zero mean data sequence. We assume $t_{k}$ and $d_{k}$ have unit powers, i.e., $\mathrm{E}\left\{t_{k}^{2}\right\}=\mathrm{E}\left\{d_{k}^{2}\right\}=1$. Also, we denote $\rho_{t}$ and $\rho_{d}$ as the pilot and data power allocation coefficients, respectively. 
Under superimposed training, $h_{k}$ and $\left(y_{k}, y_{k-1}, \cdots\right)$ are not jointly Gaussian. Therefore, the MMSE estimator is difficult to implement thus intractable. We choose instead the linear MMSE (LMMSE) channel estimator implemented by the Kalman filter. Let $\hat{h}_{k}$ be the LMMSE estimator of $h_{k}$ based on current and all the past observations $\left\{y_{k}, y_{k-1}, \cdots\right\}$, then the Kalman filter can be again used as the optimal LMMSE estimator to track the channel. Let $M[k] \triangleq \mathrm{E}\left\{\left|h_{k}-\hat{h}_{k}\right|^{2}\right\}$. Define the steady-state MSE $\mathcal{E}^{\text {sup } \triangleq} \lim _{k \rightarrow \infty} M[k]$, then solving the steady-state Riccati equation of the channel MMSE under the Kalman update, we have

$$
\mathcal{E}^{\text {sup }}=\frac{\sigma_{h}^{2}}{\frac{1}{2}(1+\kappa)+\sqrt{\left[\frac{1}{2}(1+\kappa)\right]^{2}+\frac{a^{2}}{1-a^{2}} \kappa}} .
$$

where $\kappa \triangleq \sigma_{h}^{2} \rho_{t}^{2} /\left(\sigma_{h}^{2} \rho_{d}^{2}+\sigma_{w}^{2}\right)$ is the received signal-to-interference plus noise ratio. Note that at the steady-state, in contrast to the TDM training scheme, the channel MSE in this case is time-invariant.

We again consider BPSK signaling. The detector detects $d_{k}$ based on $\hat{h}_{k}$ and $y_{k}$ by

$$
\hat{d}_{k}=\operatorname{sign}\left\{\operatorname{Re}\left\{\hat{h}_{k}^{*}\left(y_{k}-\rho_{t} t_{k} \hat{h}_{k}\right)\right\}\right\} .
$$

Notice that this detector is not the true ML detector based on $y_{k}$ and $\hat{h}_{k}$; it is the pseudo ML that assumes the estimated $\hat{h}_{k}$ has no error. At the steady state, we obtain the bit error probability by

$$
\begin{aligned}
P_{e}= & \mathrm{E}\left\{\operatorname{Pr}\left(\hat{d}_{k} \neq d_{k} \mid d_{k}\right)\right\} \\
= & \frac{1}{2}-\frac{1}{4} \frac{g+\frac{\rho_{d}}{\rho_{t}}\left(1-\frac{\mathcal{E}^{\text {sup }}}{\sigma_{h}^{2}}\right)}{\sqrt{\left[\frac{1}{\kappa}+\left(1+2 \frac{\rho_{d}}{\rho_{t}}\right) \frac{\mathcal{E}^{\text {sup }}}{\sigma_{h}^{2}}-2 g\right]\left(1-\frac{\mathcal{E}^{\text {sup }}}{\sigma_{h}^{2}}\right)}} \\
& -\frac{1}{4} \frac{g-\frac{\rho_{d}}{\rho_{t}}\left(1-\frac{\mathcal{E}^{\text {sup }}}{\sigma_{h}^{2}}\right)}{\sqrt{\left[\frac{1}{\kappa}+\left(1-2 \frac{\rho_{d}}{\rho_{t}}\right) \frac{\mathcal{E}^{\text {sup }}}{\sigma_{h}^{2}}-2 g\right]\left(1-\frac{\mathcal{E}^{\text {sup }}}{\sigma_{h}^{2}}\right)}} .
\end{aligned}
$$

where

$$
g \triangleq \frac{\left(\frac{a^{2}}{1-a^{2}} \frac{\mathcal{E}^{\text {sup }}}{\sigma_{h}^{2}}+1\right)}{\left(\frac{a^{2}}{1-a^{2}} \frac{\mathcal{E}^{\text {sup }}}{\sigma_{h}^{2}}+1\right)+\frac{1}{\left(1-a^{2}\right) \kappa}}\left(\frac{1}{\kappa}-\frac{\rho_{d}^{2}}{\rho_{t}^{2}}\right) .
$$

\section{PERFORMANCE COMPARISON UNDER TDM AND SUPERIMPOSED SCHEMES}

We compare the optimal TDM training (RPP-1) with the superimposed training under the same power constraint. We hence need to impose that both the transmission power $P$ used and the ratio of power allocated to pilots and data in each scheme are the same.

1) Optimal vs. Suboptimal TDM Schemes: We compare the performance under different TDM RPP- $\gamma$ schemes. The received signal-to-noise ratio is defined by $\mathrm{SNR}=\sigma_{h}^{2} P / \sigma_{w}^{2}$. Fig. 3 (a) shows the maximum steady-state MMSE vs. $a$ for $\mathrm{SNR}=20 \mathrm{~dB}$, and (b) shows the maximum steady-state BER performance vs. SNR at $a=0.985$. We set $\eta=20 \%$ and $\sigma_{d}^{2}=\sigma_{p}^{2}$. From (a), we observe that the largest gain obtained by placing pilot symbols optimally is when $a$ was in the range from 0.9 to 1 , which is a common range of channel time variation. From (b) we notice that the gain of the optimal placement increased with SNR, and placing pilot symbols optimally could result in several $\mathrm{dBs}$ gain and achieve a much lower error floor.

2) Superimposed vs. RPP-1 Schemes: Fig. 4(a) and (b) show the BER performance vs. fading rate $a$ for superimposed schemes and RPP- 1 with $\eta=10 \%$ pilots under $\mathrm{SNR}=20 \mathrm{~dB}$ and $5 \mathrm{~dB}$, respectively. We set half of the transmission power to pilot symbols, i.e., $\rho_{d}^{2}=\rho_{t}^{2}$. Average BER is also shown for the RPP-1 scheme. For high SNR (20dB), we observe in Fig. 4(a) that RPP-1 performs better than the superimposed scheme for slowly varying channels ( $a$ above 0.98). For such cases, the TDM scheme gives more accurate channel estimates during training than the superimposed training. We will see, however, that as channel varied more rapidly, the TDM training deteriorated, at also a more rapid rate than that of the superimposed scheme. It is apparent that even for the common fade rates at $0.9 \leq a \leq 0.97$, the superimposed scheme which offers better tracking was preferred. The advantage of the superimposed training was more pronounced when SNR was lowered to $5 \mathrm{~dB}$ as shown in Fig. 4(b). Fig. 5 (a) and (b) show the BER performance under variation of SNR for $a=0.99$ and $a=0.95$, respectively. Similar performance gain regime for each scheme can be seen. For $a=0.99$ (very slow variation), at low SNR, we see that there is little difference of the performance under the two types of schemes. At high SNR, RPP-1 provides better performance. For $a=0.95$, however, we see that the superimposed scheme uniformly performs better than RPP-1 at different SNR, even when comparing the average BER performance.

\section{CONCLUSION}

In this paper, we have studied two different forms of training schemes using the MMSE of the channel estimation and BER as the figuresof-merit. For the Gauss-Markov fading channels, we have established the optimality of RPP-1 among all periodic TDM schemes. The optimality of RPP-1 holds uniformly across all SNR levels and all fade rates. This results allows us to compare the best TDM training with the superimposed training. We show that, while the traditional TDM training performs better for slow fading channels at high SNR, the superimposed scheme outperforms the the best TDM scheme in other regimes of practical importance.

\section{REFERENCES}

[1] S. Adireddy, L.Tong, and H.Viswanathan. "Optimal Placement of Training for Frequency Selective Block-Fading Channels," IEEE Trans. on Info. Theory, 48(8):2338-2353, August 2002.

[2] C. Budianu and L. Tong. "Channel Estimation for SpaceTime Orthogonal Block Code," IEEE Trans. on Signal Processing, 48(10):2515-2528, October 2002.

[3] James K. Cavers. "An Analysis of Pilot Symbol Assisted Modulation for Rayleigh Fading Channels," IEEE Trans. on Veh. Tech., 40:686-693, November 1991.

[4] M. Dong, S.Adireddy, and L. Tong. "Optimal Pilot Placement for Semi-Blind Channel Tracking of Packetized Transmission over Time-Varying Channels," IEICE Transactions on Fundamentals of Electronics, Communications and Computer Sciences, E86-A(3), March 2003.

[5] M. Dong and L. Tong. "Optimal Design and Placement of Pilot Symbols for Channel Estimation,” IEEE Trans. on Signal Processing, 50(12):3055-3069, December 2002. 
[6] R.A. Iltis. "Joint Estimation of PN Code Delay and Multipath Using the Extended Kalman Filter," IEEE Trans. Commun., 38(10):1677-1685, October 1990.

[7] M. Médard. "The Effect upon Channel Capacity in Wireless Communications of Perfect and Imperfect Knowledge of the Channel," IEEE Trans. Information Theory, 46(5):933-946, May 2000.

[8] M. Médard, I. Abou-Faycal, and U. Madhowf. "Adaptive Coding with Pilot Signals," In 38th Allerton Conference, October 2000.

[9] R. Negi and J. Cioffi. "Pilot Tone Selection For Channel Estimation In A Mobile OFDM System," IEEE Trans. on Consumer Electronics, 44(3):1122-1128, Aug. 1998.

[10] S. Ohno and G. B. Giannakis. "Optimal training and redundant precoding for block transmissions with application to wireless OFDM,” In ICASSP, May 2001.

[11] S. Ohno and G. B. Giannakis. "Average-Rate Optimal PSAM Transmissions over Time-Selective Fading Channels," IEEE Trans. on Wireless Communications, 1(4):712-720, October 2002.

[12] M. Stojanovic, J.G. Proakis, and J.A. Catipovic. "Analysis of the Impact of Channel Estimation Errors on the Performance of a Decision-Feedback Equalizer in Fading Multipath Channels," IEEE Trans. Commun., 43:877-886, February 1995.

[13] J. M. Torrance and L. Hanzo. "Comparative study of pilot symbol assisted modem schemes," In Proc. 6th Conf. Radio Receivers and Associated Systems, , pages 36-41, Sept. 1995.
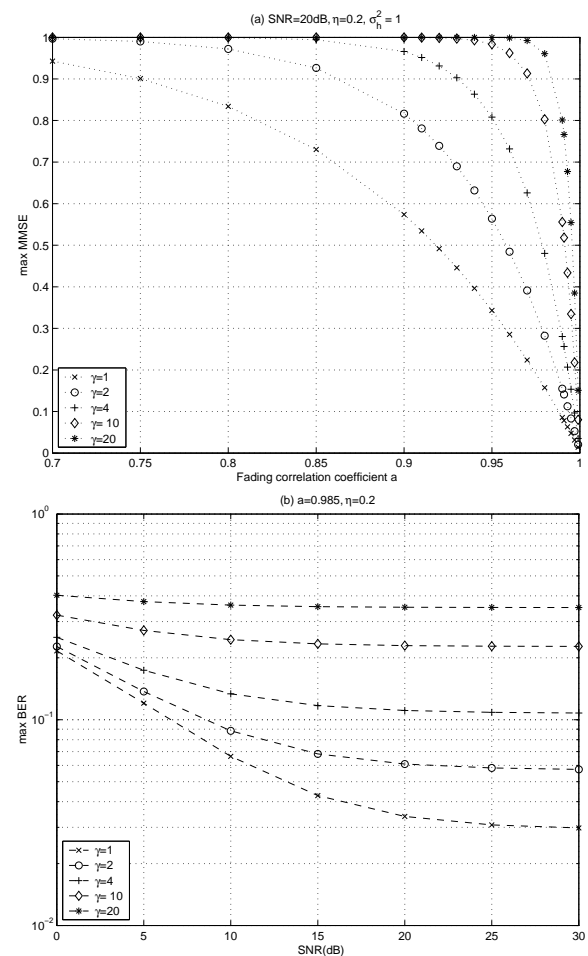

Fig. 3. (a) $\mathcal{E}(\gamma)$ vs. $a(\mathrm{SNR}=20 \mathrm{~dB})$; (b) Max. BER vs. SNR. $(a=0.985)$
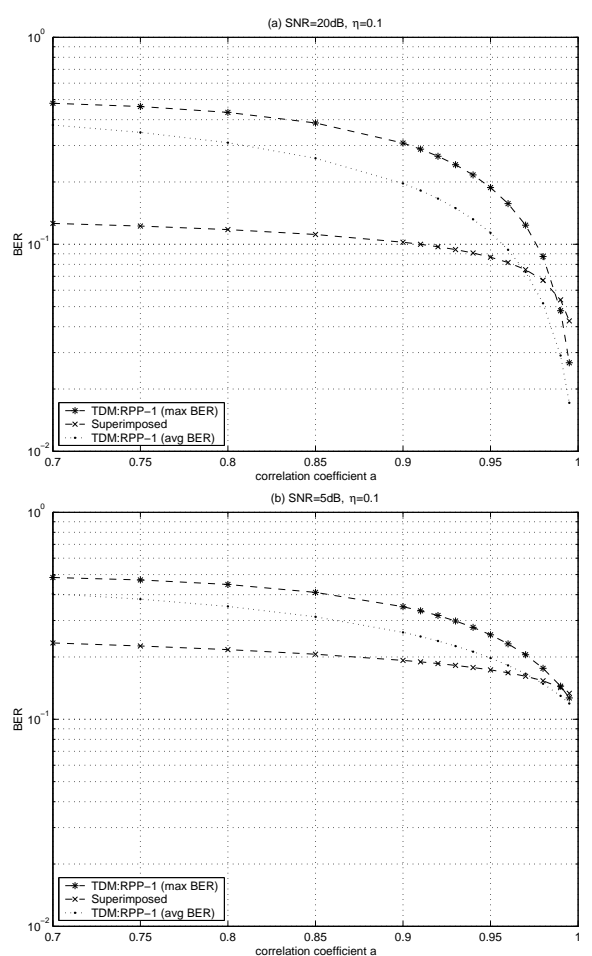

Fig. 4. Max. steady-state BER vs. a. $\quad(\eta=10 \%)$. (a) $\mathrm{SNR}=20 \mathrm{~dB}$; (b) $\mathrm{SNR}=5 \mathrm{~dB}$
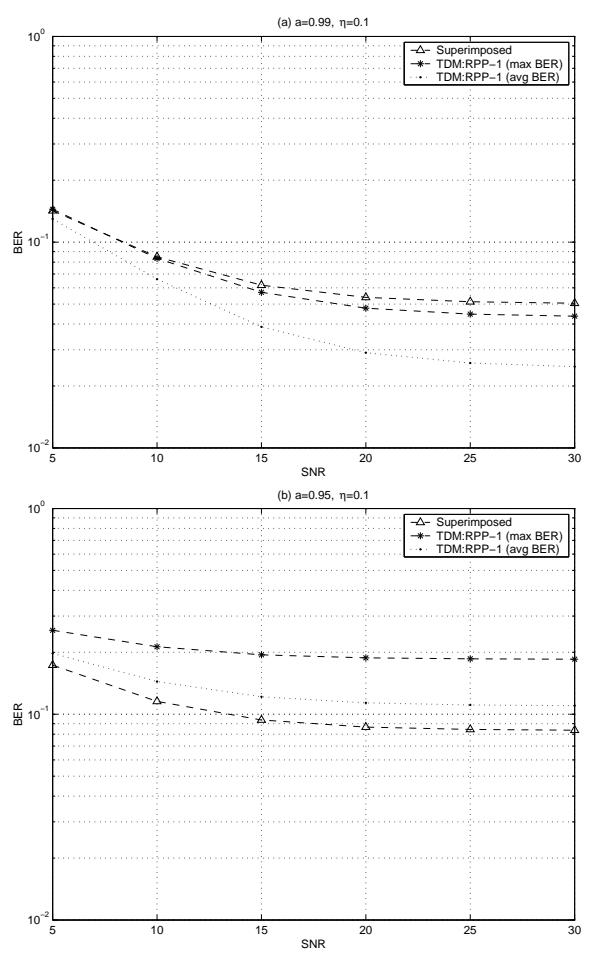

Fig. 5. (a) Max. steady-state BER vs. SNR ( $a=0.99, \eta=10 \%)$; (b) Max. steady-state BER vs. SNR. $(\mathrm{a}=0.95, \eta=10 \%)$ 